\title{
An Improved Mechanism for Congestion Control in TCP for Ad Hoc Network
}

\author{
Mukesh Kumar Dhariwal \\ School of Information \\ Technology \\ Rajiv Gandhi Proudyogiki \\ Vishwavidyalaya, BHOPAL
}

\author{
Sanjeev Sharma \\ School of Information \\ Technology Rajiv Gandhi \\ Proudyogiki Vishwavidyalaya, \\ BHOPAL
}

\begin{abstract}
Mobile Ad Hoc networks are collections of mobile nodes, dynamically forming a temporary network without pre-existing network infrastructure or centralized administration. Transmission control protocol (TCP) provides connection oriented, reliable and end to end mechanism. Comparing to wire networks, there are many different characteristics in wireless environments.

In this paper an improved mechanism for TCP congestion control is presented. It considers the influences sending rate to TCP sender's packet only by the congestion. The drop rate for TCPM is calculated according to total packet received and total packet acknowledgment in network. So, there is less packet drop in transmission. The comparative study of Modified TCP (TCPM) with other TCP variants is also carried out on various parameters like pause time, size of network. The improved mechanism was implemented with fewer overheads and effectively improves reliability with small variances of throughput and delay. Implementations and Simulations were performed in QualNet 5.0 simulator.
\end{abstract}

\section{Keywords}

Wireless network, congestion control, packet loss rate, TCP variants.

\section{INTRODUCTION}

Transmission control protocol (TCP) [1] [2] is the predominant Internet protocol and carries approximately $90 \%$ of Internet traffic in today's heterogeneous wireless and wired networks. TCP is widely used as a connection oriented transport layer protocol that provides reliable packet delivery over unreliable links. TCP does not depend on the underlying network layers and, hence, design of various TCP variants is based on the properties of wired networks. However, TCP congestion control algorithms may not perform efficiently in heterogeneous networks.

Wireless networks have higher bit error rates due to weather conditions, obstacles, multipath interferences, mobility of wireless end-devices, signal attenuation and fading, which may lead to packet loss. Various TCP algorithms and techniques have been proposed to improve congestion and reduce the noncongestion related packet loss. TCP Tahoe [12], TCP Reno [12] with Selective Acknowledgement (SACK) [12], TCP New Reno [13], TCP Vegas [16], and TCP FACK [15] are examples of previously proposed end-to-end solutions and for improving network performance [3] [9]. The end-to-end techniques are the most promising because they require changes only to the end systems rather than to the intermediate nodes. These end-to-end control approaches are used in today's deployed networks.

In Section 2, algorithm of TCPM is described. Description of simulated network is given in Section 3, while simulation scenarios and results are described in Section 4 and 5, conclusion is described in Section 6 and future work is explained with Section 7.

\subsection{Packet loss Rate}

In wireless mobile networks, usually loss of packets is because of congestion of data on the wireless link. Wireless networks are characterized by large error rates due to fading, noise, interference from other sources and mobile host movement. Due to large error rate, packet losses due to congestion in the wireless link are more as compared to congestion losses. The packet loss rate depends on lost packet it will be occur due to congestion. Packet drop rate is not adjusted when the congestion loss rate is low, but it is required to decrease the drop rate rapidly to improve the reliability when the congestion loss rate becomes higher. Otherwise, there will be more lost packets due to congestion and more packets will be retransmission, responding the poor transmission reliability and more energy consumption of mobile hosts.

Total drop rate is denoted by $\mathrm{Td}[10]$. If sender sends $\mathrm{n}$ packets in a time period $\mathrm{T}$ and $\mathrm{m}$ packets of them be discard because one or more bits error caused by wireless link congestion, the total drop rate is define as

$$
\mathbf{T}_{\mathrm{d}}=\mathbf{T}_{\mathrm{r}}-\mathbf{T}_{\mathrm{a}}
$$

\section{MODIFIED TCP}

Modified Transport Control Protocol (TCPM) is the improved TCP congestion control mechanism for wireless network. It considers the influences to TCP sender's packet sending rate only by the congestion but also by the link failure. It is a good reference to apply the TCP to wireless networks.

\subsection{Initial Window}

The initial sending window is calculated by following formula: $I w=\min (4 * S M S S, \operatorname{Max}(2 * S M S S, 4380$ byte $))$;

\subsection{Slow-Start Algorithm}

The slow start algorithm is used to start a connection of TCPM and the periods after the value of retransmission timer exceed the RTO (retransmission timeout). In the start of TCPM, the size 
of cwnd will be initialized to 1 .The slow start algorithm describes as below:

if (Receive ACKs \&\& cwnd < ssthresh)

cwnd $=$ cwnd $++;\}$

The slow start algorithm will be ended in two conditions. First, if the congestion window size reaches the slow start threshold size (ssthresh), the slow start will be ended and then congestion avoidance takes over. Second, if there lose any packet due to congestion or high packet loss rate due to corruption, the slow start also will be ended and then fast recovery takes over.

\subsection{Congestion Avoidance Algorithm}

If the congestion window size (cwnd) is less than or equal to the slow start threshold size (ssthresh), TCPM is in slow start; otherwise TCPM is performing congestion avoidance. The congestion avoidance algorithm describes as below:

if (Receive ACKs \|| (Receive Explicit Corruption Loss

Notification \&\& Corruption Loss Rate Pe $<$ Pemin) $)\{$

if (cwnd > ssthresh)

cwnd $=1+1 /$ cwnd

else

cwnd++;

$\mathrm{T}_{\mathrm{d}}=\mathrm{T}_{\mathrm{r}}-\mathrm{T}_{\mathrm{a}}$

If $\left(\mathrm{T}_{\mathrm{d}}>10 \%\right)$

Go to slow start algorithm \}

Where,

$\mathrm{T}_{\mathrm{d}}=$ Total drop

$\mathrm{T}_{\mathrm{r}}=$ Total Received

$\mathrm{T}_{\mathrm{a}}=$ Total acknowledge

In the algorithm, cwnd denotes the congestion window size.

\subsection{Fast Retransmission and Fast Recovery}

If the network congestion or heavy corruption, the fast recovery algorithm will be taken. When the network congestion, set ssthresh to one-half the flight size or double of MSS (maximum segment size) window.

if (Congestion $\|$ Heavy Corruption) \{

if (Receive Same ACK 3 Times $\|$ Retransmission

Timer Overtime) $/ *$ Congestion $* /\{$

Ssthresh $=\max ($ flightsize $/ 2,2 *$ SMSS $) ; / /$ Flightsize are those data which have no acknowledged.

if (Retransmission Timer Overtime)

\{cwnd $=1$; Exit and call slow-start; $\}$

else /* Receive Same ACK 3 Time */

cwnd $=$ ssthresh; $\}$

else if $\left(\mathrm{T}_{\mathrm{d}}>10 \%\right)$

Go to slow start algorithm

\section{COMPUTER SIMULATION}

All the simulations were performed in QualNet [11] wireless network simulator version 5.0. Initially number of nodes are 50, Simulation time was taken 200 seconds and seed as 1 . All the scenarios have been designed in $1500 \mathrm{~m} \times 1500 \mathrm{~m}$ area. Mobility model used is Random Way Point (RWP). In this model a mobile node is initially placed in a random location in the simulation area, and then moved in a randomly chosen direction. The movement proceeds for a specific amount of time or distance, and the process is repeated a predetermined number of times. Number of nodes in network 10 to 50 and with pause time of $5 \mathrm{~s}$ to $30 \mathrm{~s}$.

All the simulation works was carried out using TCP variants (Reno, Tahoe) with DSR routing protocol .Network traffic is provided by using File Transfer Protocol (FTP) application. File Transfer Protocol (FTP) represents the File Transfer Protocol server and client.

\section{PERFORMANCE METRICS USED FOR THIS WORKS ARE AS FOLLOWS}

4.1 Throughput is the measure of the number of packets successfully transmitted to their final destination per unit time. It is the ratio between the numbers of sent packets vs. received packets.

4.2 Signal Received with error is the measure of signal received, but they have error. The error may be occurring due to noise or due to heavy traffic.

4.3 Bytes Received are the measure of total packet received by server. The packets may be drop due to heavy traffic. So received packets may be vary according to traffic conditions.

4.4 Packet loss is the measure of total discarded packet due to corruption or due to packet drop. It can be calculate by subtracting total received packets by server with total sent packet by client.

\section{RESULTS AND ANALYSIS}

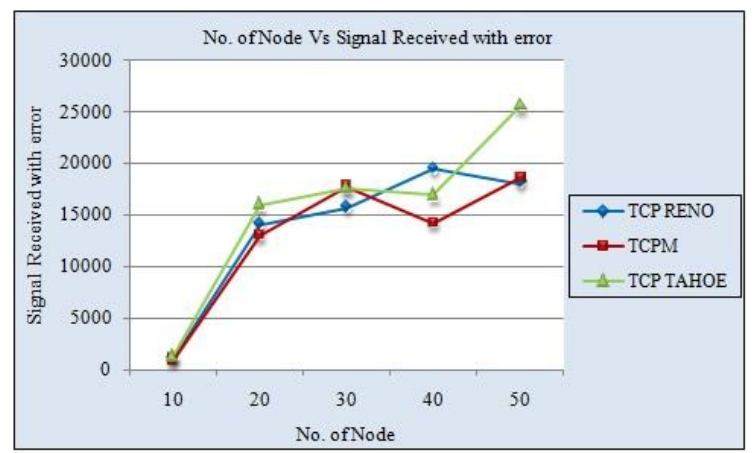

Fig. 1

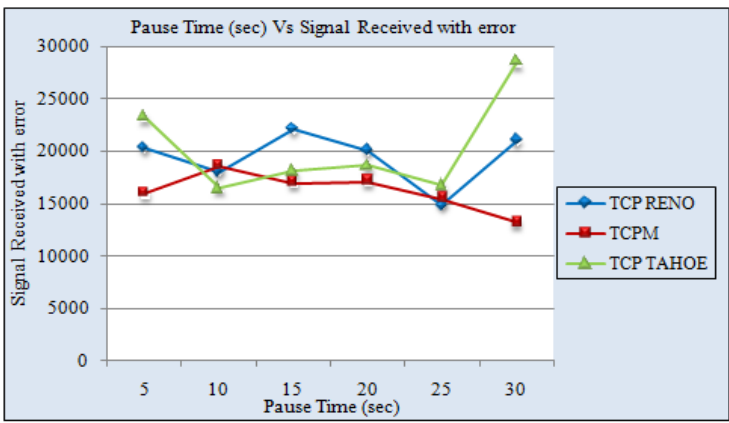

Fig. 2 
Fig $1 \& 2$ shows signal received with error of different variants with pause time and number of node in network. It is observed that TCPM has less error signal than other TCP variants, because of optimal routing path between sender and receiver. Increment in the no. of nodes causes congestion consequently signal were distorted.

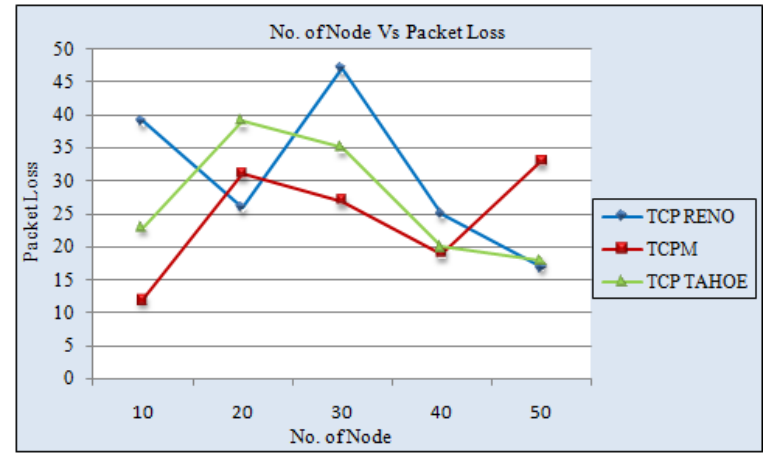

Fig. 3

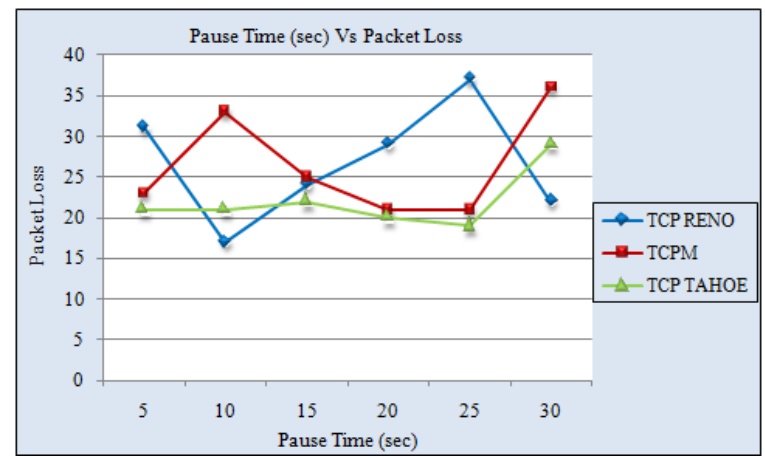

Fig.4

Fig $3 \& 4$ shows Packet loss of different variants of TCP, pause time and number of node respectively. It is observed that TCPM has less packet loss than other TCP variants. Because of proper sending window size, this is calculated according to number of discarded packets. Therefore, receiver can receive approximately all packets which are sent by sender.

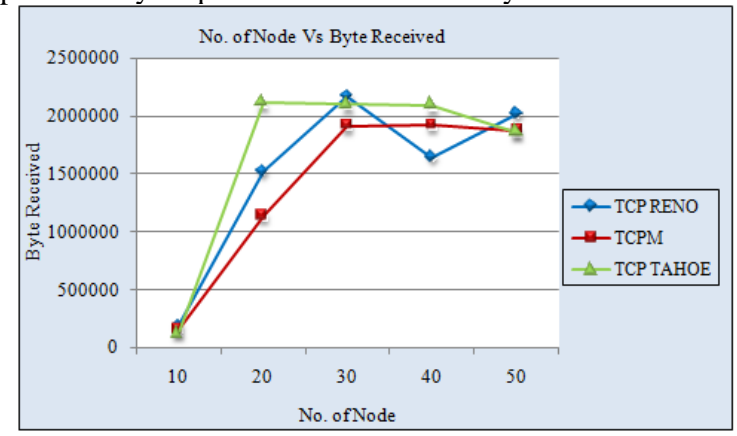

Fig. 5

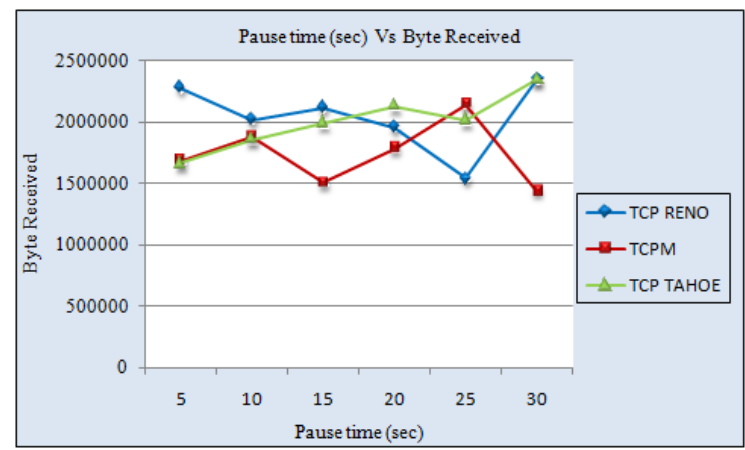

Fig. 6

Fig 5 \& 6 shows the readings of total byte received for different TCP variants, pause time and number of node in network respectively. It is observed that receiver can receive maximum byte at TCPM, except under congestion in network. Sometimes nodes move faster, hence it cannot get proper signal, consequently loss of packets.

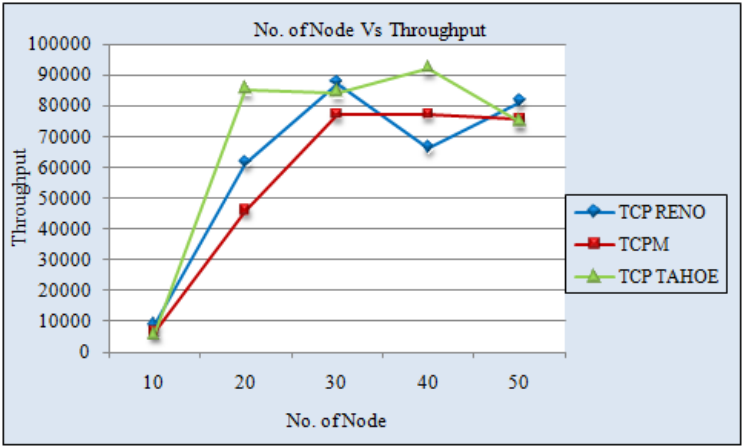

Fig.7

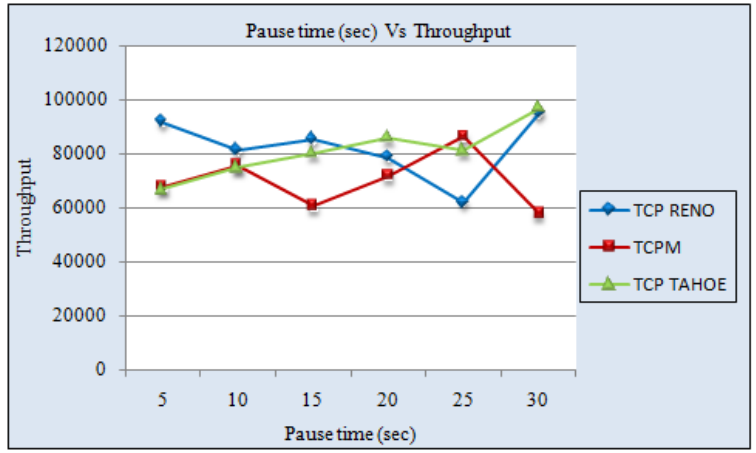

Fig.8

Fig $7 \& 8$ shows throughput of different TCP variants with pause time and number of node. Throughput is the ratio of numbers of sent packets with number of received packets. It is also observed that throughput of TCPM is better than other TCP variants. Here receiver receives maximum packets and only fewer packets were discarded, so we can conclude that here in TCPM throughput is a better then other TCP variant. 


\section{CONCLUSION}

A modified TCP (TCPM) is proposed with a new calculation for packet drop and implemented it in a Mobile Ad-hoc Network in QualNet 5.0. It considers the influences to TCP sender's packet sending rate only by the congestion. The calculation of packet drop for TCPM is based on total packet received and total packet acknowledgment in network.

Extensive simulation were undertaken to compare its performance with other standards TCP Reno, and TCP Tahoe over Ad-hoc Mobile Network. The simulation shows that the performance of TCPM is better than other TCP variants. The performance of TCP variants are analyzed on various parameters like pause time, size of network. From results of implementation it found that the performance of TCPM is better in high density node as in this circumstance sender can get different paths through different nodes. Packets can be dropped due to congestion in network or heavy traffic, so that packet drop is fewer in less density network. Throughput depends on number of packet received and number of packets loss. It is better in high density network, because receiver can receive maximum packets when number of node in network is 50. Throughput is also better when node in network will be increases and it is also better when node moves with pause time of $30 \mathrm{sec}$.

\section{FUTURE WORK}

The performance of TCPM can be measured in different wireless environments especially those with high error rates. Plan to build a flexible and lightweight transport protocol for the wireless side of TCPM, which can adopt changes in the wireless environment and can support planned disconnections seems to be a high potential in future.

\section{REFERENCES}

[1] J. Postel, "Transmission control protocol," RFC 793, Sept.1981.

[2] A. Gurtov and S. Floyd, "Modeling wireless links for transport protocols," ACM SIGCOMM Comput. Commun. Rev., vol. 34, no. 2, pp. 85-96, Apr. 2004.

[3] G. Holland and N. Vaidya, "Analysis of TCP performance over mobile ad hoc networks," in Proc. ACM/IEEE Int. Conf. on Mobile Computing, Seattle, WA, USA, Sept. 1999, pp. 219-230.

[4] Y. Shang and M. Hadjitheodosiou, "TCP splitting protocol for broadband and aeronautical satellite network," in Proc.23rd IEEE Digital Avionics Syst. Conf., Salt Lake City, UT, Oct. 2004, vol. 2, pp. 11.C.3-1-11.C.3-9.

[5] J. Zhu, S. Roy, and J. H. Kim, "Performance modeling of TCP enhancements in terrestrial-satellite hybrid networks," IEEE/ACM Trans.Netw., vol. 14, no. 4, pp. 753-766, Aug. 2006.

[6] C.-H. Ng, J. Chow, and Lj. Trajkovic, "Performance evaluation of the TCP over WLAN 802.11 with the Snoop performance enhancing proxy," OPNETWORK 2002, Washington, DC, Aug. 2002.

[7] W. G. Zeng, M. Zhan, Z. Li, and Lj. Trajkovic, "Improving TCP performance with periodic disconnections over wireless links," OPNETWORK 2003, Washington, DC, Aug. 2003.

[8] W. G. Zeng and Lj. Trajkovic, "TCP packet control for wireless networks," in Proc. IEEE Int. Conf. on Wireless and Mobile Computing, Networking and Communications (WiMob 2005), Montreal, Canada, Aug. 2005, vol. 2, pp. 196-203.

[9] M. Omueti and Lj. Trajkovic, "M-TCP+: using disconnection feedback to improve performance of TCP in wired/wireless networks," in Proc. SPECTS 2007, San Diego, CA, USA, July 2007, pp. 443-450.

[10] Xu Chang-Biao, Long Ke-Ping, Yang Shi Zhong. Corruption-based TCP rate adjustment in wireless networks. In: Chinese Journal of Computers, 2002, 25(4): pp.438-444.

[11] Scalable Network Technology, "QualNet4.0 simulator" tutorial and QualNet Forum, http://www.scalablenetworks.com/forums/

[12] K.Fall, S.Floyd "Simulation Based Comparison of Tahoe, Reno and SACK TCP".

[13] S. Floyd,The NewReno Modification to TCP's Fast Recovery Algorithm, RFC 3782, April 2004.

[14] B. Qureshi, M. Othman, Member, IEEE, and N. A. W. Hamid "Progress in Various TCP Variants" February 2009.

[15] M. Mathis and J. Mahdavi, "Forward acknowledgment: refining TCP congestion control" in Proceedings of ACM SIGCOMM, pp. 181-191, 1996.

[16] Lawrence S. Brakmo, Student Member, IEEE, and Larry L. Peterson TCP Vegas: End to End Congestion Avoidance on a Global Internet, OCTOBER 1995. 